УДК 597.554.3, 574.24

doi: $10.17223 / 19988591 / 41 / 9$

\title{
Н.О. Яблоков
}

Научно-исследовательский институт экологии рыбохозяйственных водоемов, г. Красноярск, Россия

\section{Морфологические аномалии скелета у молоди рыб р. Качи (бассейн Среднего Енисея) в градиенте техногенной нагрузки}

\begin{abstract}
Представлены данные о разнообразии и встречаемости морфологических аномалий в развитии скелета у молоди пескаря сибирского Gobio gobio cynocephalus Dybowski, 1869 и гольяна речного Phoxinus phoxinus (L., 1758) для четырех участков р. Качи (бассейн Среднего Енисея). В общей сложности диагностировано 95 аномалий морфологии скелета, принадлежсащих $\kappa$ 12 типам и локализованных в 6 отделах скелета. У молоди пескаря обнаружень 62 аномалии 7 типов, у молоди гольяна - 33 аномалии 10 типов. Отмеченные аномалии локализовались преимущественно в хвостовом отделе позвоночника и представляли собой разнообразные деформации невральных и гемальных дуг, такие как незамкнутые дуги позвонков, сращение дуг разных позвонков, перемещение дуг на соседние позвонки. Встречаемость аномалий варьировала в пределах от 6,5 до 55,7\% у молоди пескаря, от 8,4 до 75,0\% у молоди гольяна. Частота встречаемости аномальных особей составила 4,6-10,4\% для молоди пескаря и 7,4-11,7\% для молоди гольяна. Доли тяжельх форм аномалий у молоди двух видов были близки и изменялись в пределах от 0 до 80\% от верхнего течения реки к нижнему. При анализе встречаемости аномалий в р. Каче прослеживается тенденция увеличения частоты встречаемости по мере усиления антропогенной нагрузки на водоток. В иелом значения показателей встречаемости аномалий $в$ развитии скелета в нижнем течении р. Качи близки к частотам встречаемости аномалий позвоночного столба у молоди рыб, ранее отмеченным в ряде пригородных водотоков г. Красноярска.
\end{abstract}

Ключевые слова: онтогенез рыб; морфологические аномалии; техногенное воздействие; Phoxinus phoxinus; Gobio gobio cynocephalus.

\section{Введение}

Преобладающая часть малых рек России подвержена активному техногенному воздействию [1]. Наиболее критично это проявляется в городских и пригородных водных объектах, принимающих на себя огромное количество поллютантов органического и неорганического происхождения [2]. В бассейне р. Енисей ярким примером такого воздействия является р. Кача (левобережный приток первого порядка протяженностью 102 км), нижнее течение которой находится в черте г. Красноярска и подвергается постоян- 
ному воздействию техногенных загрязнителей. Согласно Государственному докладу «О состоянии и об охране окружающей среды Российской Федерации в 2014 году» участок реки, расположенный в черте г. Красноярска, внесен в перечень наиболее загрязненных водных объектов на территории РФ [3]. Список поллютантов в р. Каче включает железо, медь, цинк, алюминий, марганец и фенолы [4]. В 2015 г. нормативы качества превышали среднегодовые значения для следующих показателей: ХПК (в 1,2 раза), БПК 1,1 раза), железо общее (4,3 ПДК), алюминий (12 ПДК), марганец (9 ПДК), медь (2,5 ПДК) [5]. На протяжении нескольких лет в реке отмечается тенденция увеличения среднегодовых концентраций металлов (алюминия, марганца, железа) в направлении от истока к устью.

При определении экологического состояния водных экосистем широко применяется индикация по состоянию организмов гидробионтов [6-7]. Одну из важнейших позиций здесь занимает рыбное сообщество. Это связано с высоким разнообразием занимаемых рыбами экологических ниш, продолжительным жизненным циклом, главенствующим положением в пищевых цепях и рядом других факторов [8-10]. Рыбы наиболее чувствительны к воздействию поллютантов главным образом на ранних стадиях онтогенеза, в связи с наименьшим уровнем токсикорезистентности по сравнению с взрослыми особями, а также невозможностью активно уходить из районов с высоким уровнем загрязнения [9]. На основании этого ряд авторов в качестве критерия оценки качества вод предлагают использовать показатели разнообразия и встречаемости морфологических аномалий развития личинок и молоди рыб, являющихся своеобразным ответом на действие комплекса неблагоприятных факторов абиотического происхождения, в том числе присутствие в экосистеме токсических веществ [10-13]. Несмотря на обилие натурных и экспериментальных исследований, посвященных изучению морфофункциональных нарушений, возникающих у рыб, населяющих водные объекты с различной степенью антропогенной нагрузки, аспекты изучения механизмов возникновения морфологических аномалий в настоящее время не теряют своей актуальности и играют важную роль в оценке состояния популяций рыб [13-14].

Цель настоящей работы - изучение разнообразия и встречаемости аномалий развития скелета у молоди гольяна речного и пескаря сибирского в р. Каче.

\section{Материалы и методики исследования}

Объектом исследования являлась разновозрастная молодь гольяна речного Phoxinus phoxinus (L., 1758) и пескаря сибирского Gobio gobio cynocephalus Dybowski, 1869 - наиболее распространенных и многочисленных видов рыб, населяющих бассейн р. Енисей.

Ихтиологический материал отбирался в июле-августе 2016 г. на четырех участках реки, различающихся уровнем техногенной нагрузки и спектром 
загрязняющих веществ: станция 1 - в черте г. Красноярска; станция 2 д. Дрокино, 1 км ниже деревни; станция 3 - пос. Памяти 13 Борцов, 1 км ниже поселка; Станция 4 - пл. Лесная, 0,5 км ниже ж/д полотна (рис. 1). Перечень основных загрязняющих агентов, а также сводная информация по результатам мониторинга окружающей среды на исследуемых участках реки приведены в табл. 1.

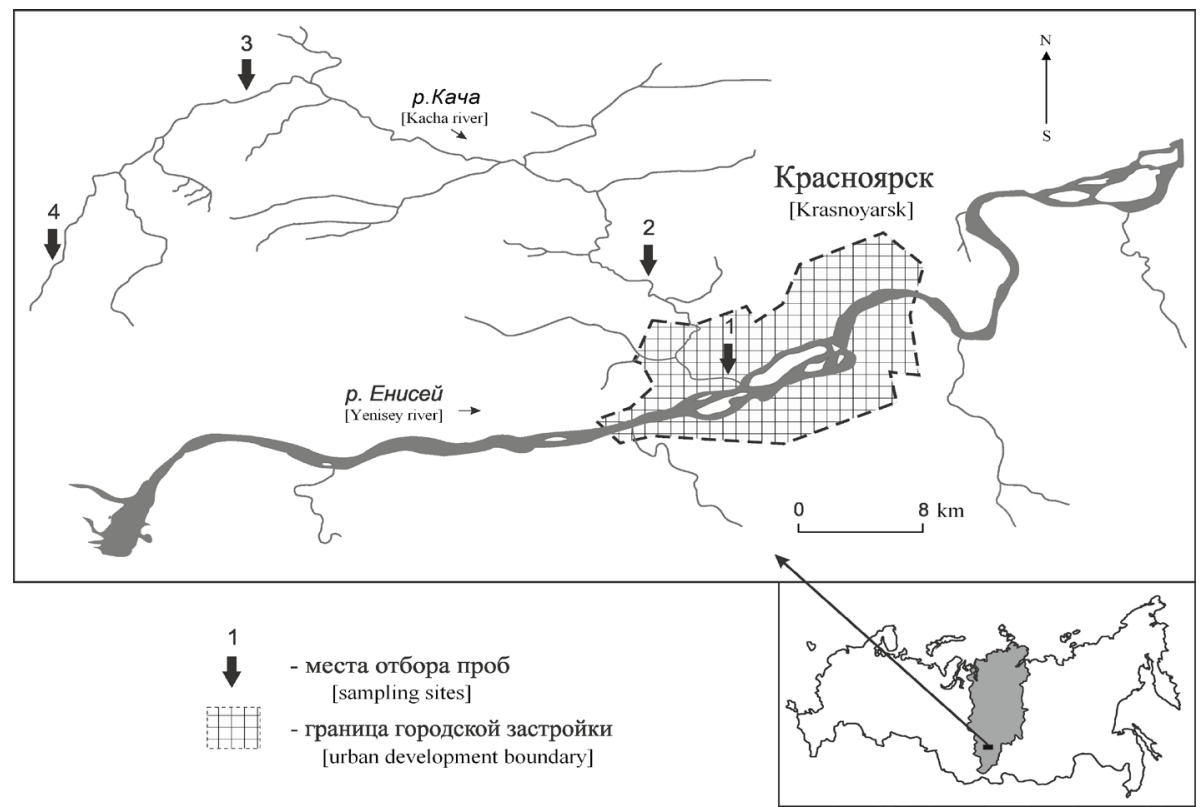

Рис. 1. Карта-схема района исследований

[Fig. 1. Schematic map of the study area]

Т а б л и ц а 1 [Table 1]

Данные мониторинговых исследований в пределах участков отбора проб [Results of monitoring studies at the sampling sites]

\begin{tabular}{|c|c|c|c|c|c|c|}
\hline № & $\begin{array}{l}\text { Место отбора } \\
\text { [Sampling site] }\end{array}$ & \begin{tabular}{|c} 
Рассто- \\
яние от \\
устья, км \\
[Distance \\
from the \\
river mouth, \\
km] \\
\end{tabular} & $\begin{array}{c}\text { Коорди- } \\
\text { наты } \\
\text { [Coordi- } \\
\text { nates] }\end{array}$ & $\begin{array}{c}\text { УКИЗВ } \\
\text { [YuКІZV] } \\
{[5]}\end{array}$ & $\begin{array}{c}\text { Индекс } \\
\text { Вудивисса } \\
\text { [Woodiviss } \\
\text { biotic index] } \\
{[15,16]}\end{array}$ & $\begin{array}{c}\text { Загрязняющий } \\
\left.\text { агент (ПДК }{ }_{\text {сан }}\right) \\
\text { [Pollutant } \\
\left.\left(\mathrm{TLV}_{\mathrm{san}}\right)\right][5]\end{array}$ \\
\hline 1 & $\begin{array}{l}\text { Красноярск } \\
\text { [Krasnoyarsk] }\end{array}$ & 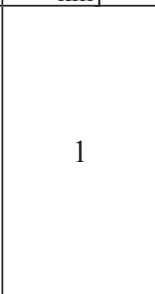 & $\begin{array}{l}56^{\circ} 01^{\prime} 06^{\prime \prime} \mathrm{N} \\
92^{\circ} 52^{\prime} 44^{\prime \prime} \mathrm{E}\end{array}$ & $\begin{array}{c}\text { 4a, грязная } \\
\text { [4a, dirty] }\end{array}$ & $\begin{array}{c}\text { V грязная } \\
\text { [V dirty] }\end{array}$ & $\begin{array}{c}\mathrm{Al}(16,4), \\
\mathrm{Mn}(18,9), \\
\mathrm{Fe}(19,3), \\
\text { нефтепродук- } \\
\text { ты }(10,2) \\
{[\text { Petroleum prod- }} \\
\text { ucts }(10,2)], \\
\text { фенолы [Phenols] }\end{array}$ \\
\hline
\end{tabular}


О к о н ч а н и е т а б л. 1 [Table 1 (end)]

\begin{tabular}{|c|c|c|c|c|c|c|}
\hline № & $\begin{array}{c}\text { Место отбора } \\
\text { [Sampling site] }\end{array}$ & $\begin{array}{c}\text { Рассто- } \\
\text { яние от } \\
\text { устья, км } \\
\text { [Distance } \\
\text { from the } \\
\text { river mouth, } \\
\text { km] }\end{array}$ & $\begin{array}{l}\text { Коорди- } \\
\text { наты } \\
\text { [Coordi- } \\
\text { nates] }\end{array}$ & $\begin{array}{c}\text { УКИЗВ } \\
\text { [YuKIZV] } \\
{[5]}\end{array}$ & $\begin{array}{c}\text { Индекс } \\
\text { Вудивисса } \\
\text { [Woodiviss } \\
\text { biotic index] } \\
{[15,16]}\end{array}$ & $\begin{array}{c}\text { Загрязняющий } \\
\left.\text { агент (ПДК }{ }_{\text {caн }}\right) \\
{[\text { Pollutant }} \\
\left.\left(\mathrm{TLV}_{\mathrm{san}}\right)\right][5]\end{array}$ \\
\hline 2 & $\begin{array}{l}\text { Дрокино } \\
\text { [Drokino] }\end{array}$ & 15 & $\begin{array}{l}56^{\circ} 05^{\prime} 23^{\prime \prime} \mathrm{N} \\
92^{\circ} 48^{\prime} 01^{\prime \prime} \mathrm{E}\end{array}$ & $\begin{array}{c}\text { 4a, грязная } \\
\text { [4a,dirty] }\end{array}$ & $\begin{array}{c}\text { IV загряз- } \\
\text { ненная } \\
\text { [IV polluted] }\end{array}$ & $\begin{array}{c}\operatorname{Mn}(15,0), \\
\text { Al }(13,4), \\
\text { фенолы [Phenols], } \\
\gamma \text {-гхцг }[\gamma-\mathrm{hch}]\end{array}$ \\
\hline 3 & $\begin{array}{c}\text { Памяти } \\
13 \text { Борцов } \\
\text { [Pam’yaty } \\
13 \text { Bortsov] }\end{array}$ & 65 & $\begin{array}{l}56^{\circ} 13^{\prime} 20^{\prime \prime} \mathrm{N} \\
92^{\circ} 20^{\prime} 21^{\prime \prime} \mathrm{E}\end{array}$ & $\begin{array}{c}\text { 4a, грязная } \\
{[4 \mathrm{a}, \text { dirty }]}\end{array}$ & $\begin{array}{c}\text { V грязная } \\
\text { [V dirty] }\end{array}$ & $\begin{array}{c}\mathrm{Al}(12,0) \\
\operatorname{Mn}(9,0) \\
\operatorname{Fe}(4,3) \\
\operatorname{Cu}(2,0)\end{array}$ \\
\hline 4 & $\begin{array}{c}\text { Лесная } \\
\text { [Lesnaya] }\end{array}$ & 87 & $\begin{array}{l}56^{\circ} 06^{\prime} 01^{\prime \prime} \mathrm{N}, \\
92^{\circ} 09^{\prime} 58^{\prime \prime} \mathrm{E}\end{array}$ & $\begin{array}{c}\text { 3б, очень } \\
\text { загрязненная } \\
\text { [3b, very } \\
\text { polluted] }\end{array}$ & - & $\mathrm{Al}(11,0)$ \\
\hline
\end{tabular}

Общая величина пробы составила 396 экз., среди которых пескаря сибирского - 184 экз., гольяна речного - 212 экз. Размерно-возрастные характеристики исследованных особей приведены в табл. 2.

Т а б л и ц а 2 [Table 2]

Размерно-возрастные характеристики проб молоди рыб из р. Качи

[Size and age characteristics of juvenile fish samples from the Kacha river]

\begin{tabular}{|c|l|c|c|c|}
\hline $\begin{array}{c}\text { Станция, № } \\
\text { [Station, No] }\end{array}$ & \multicolumn{1}{|c|}{$\begin{array}{c}\text { Вид } \\
\text { [Species] }\end{array}$} & $\begin{array}{c}N, \text { экз. } \\
\text { [Number, ind.] }\end{array}$ & $\begin{array}{c}\text { Абсолютная длина, мм } \\
{[\text { Total length, mm] }} \\
M \pm m\end{array}$ & $\begin{array}{c}\text { Возраст } \\
\text { [Age] }\end{array}$ \\
\hline \multirow{2}{*}{1} & $\begin{array}{l}\text { Gobio gobio } \\
\text { cynocephalus }\end{array}$ & 70 & $19,9 \pm 0,5$ & $0+, 1+$ \\
\cline { 2 - 5 } & Phoxinus phoxinus & 17 & $22,5 \pm 0,6$ & $0+, 1+$ \\
\hline \multirow{2}{*}{2} & $\begin{array}{l}\text { Gobio gobio } \\
\text { cynocephalus }\end{array}$ & 68 & $15,3 \pm 0,3$ & $0+$ \\
\cline { 2 - 5 } & Phoxinus phoxinus & 12 & $17,8 \pm 0,8$ & $0+, 1+$ \\
\hline \multirow{2}{*}{3} & $\begin{array}{l}\text { Gobio gobio } \\
\text { cynocephalus }\end{array}$ & 46 & $22,2 \pm 0,6$ & $0+, 1+$ \\
\cline { 2 - 5 } & Phoxinus phoxinus & 95 & $24,0 \pm 0,4$ & $0+, 1+$ \\
\hline \multirow{2}{*}{4} & Phoxinus phoxinus & 88 & $16,4 \pm 0,6$ & $0+$ \\
\hline
\end{tabular}

Морфологические аномалии скелета изучены на препаратах, предварительно окрашенных ализариновым красным по методике Поттхофа [17], с использованием стереоскопического микроскопа MC2-Zoom (Микромед, Россия) при оптическом увеличении 40×. Осмотр произведен последовательно в 9 отделах скелета (табл. 3). При этом осевой скелет разделен на 
четыре отдела - позвонки Веберова аппарата (первые четыре позвонка и их модифицированные элементы), туловищные и переходные позвонки (позвонки с открытой гемальной дугой, несущие ребра или незамкнутые парапофизы), хвостовые позвонки (позвонки с замкнутыми невральной и гемальной дугами и выраженными верхним и нижним остистыми отростками) и преуральные позвонки (три последних хвостовых позвонка) и уростиль [18-19]. Номенклатура и дифференцировка аномалий по степени тяжести приведены по аналогии с работами Ю.В. Чеботаревой и К. Боглионе с соавт. $[12,20]$. Для описания вида и расположения аномалии использовалась система условных обозначений, согласно которой каждому отделу скелета присваивался буквенный индекс, а конкретному виду аномалий числовой (см. табл. 3).

Т а б л и ц а 3 [Table 3]

Условные обозначения отделов скелета и типов отмеченных аномалий [Symbols of the skeleton regions and types of observed abnormalities]

\begin{tabular}{|c|c|}
\hline $\begin{array}{l}\text { Обозначение } \\
\text { [Symbol] }\end{array}$ & Отдел скелета [Skeleton region] \\
\hline $\mathrm{A}$ & Позвонки Веберова аппарата [Vertebrae of the Weberian apparatus] \\
\hline B & Туловищные и переходные позвонки [Abdominal vertebrae] \\
\hline $\mathrm{C}$ & Хвостовые позвонки [Caudal vertebrae] \\
\hline $\mathrm{D}$ & Преуральные позвонки и уростиль [Preural vertebrae and urostile] \\
\hline $\mathrm{E}$ & Лучи грудного плавника [Pectoral fin rays] \\
\hline $\mathrm{F}$ & Анальный плавник [Anal fin] \\
\hline $\mathrm{G}$ & Хвостовой плавник [Caudal fin] \\
\hline $\mathrm{H}$ & Спинной плавник [Dorsal fin] \\
\hline I & Череп [Cranium] \\
\hline $\begin{array}{c}\text { Обозначение } \\
\text { [Symbol] }\end{array}$ & $\begin{array}{ll}\text { Вид аномалии } \\
{[\text { Abnormality type }]}\end{array}$ \\
\hline 1 & Сращение центров позвонков* [Vertebral fusion]* \\
\hline 2 & Деформация центра позвонка* [Vertebral deformation]* \\
\hline 3 & Деформация невральной дуги* [Anomalous neural arch]* \\
\hline 4 & Деформация гемальной дуги* [Anomalous hemal arch]* \\
\hline 5 & $\begin{array}{l}\text { Деформация / недоразвитие луча плавника } \\
\text { [Anomalous / absent ray] }\end{array}$ \\
\hline 6 & Деформация гипуралии [Anomalous hypural] \\
\hline 7 & $\begin{array}{l}\text { Прогнатизм зубной кости («мопсовидность») } \\
\text { [Anomalous dentary (pugheadness)] }\end{array}$ \\
\hline 8 & Деформация ребер [Anomalous ribs] \\
\hline
\end{tabular}

Примечание. Курсивом выделены тяжелые формы аномалий; аномалии, отмеченные значком *, рассматривались как тяжелые, если отмечались у особи более чем один раз. [Note. Severe types of abnormalities are in italics, abnormalities marked by * were considered as severe if they were observed more than once].

Оценка количественных показателей морфологических аномалий скелета проводилась на основании частоты встречаемости аномалий в выборке (доля случаев аномального развития в выборке $N$ ), частоты встречаемости 
аномальных особей (доля аномальных особей в выборке $N$ ), общего спектра аномалий (набор различных видов аномалий, обнаруживаемых у всех особей в выборке, $S_{a p}$ ), числа аномалий на особь (количество аномалий, отмеченных у одной аномальной особи) и относительной встречаемости аномалий $\left(A_{r}\right)$. Относительную встречаемость аномалии рассчитывали как отношение общего числа аномалий определенного типа к сумме всех зарегистрированных случаев аномалий в выборке (в \%) [21]. Качественная оценка проводилась на основании учета доли тяжелых форм аномалий от их общего числа в выборке.

Статистическая обработка данных и построение графических изображений выполнены с использованием программ Microsoft Excel и PAST. Данные о размерном составе рыб и количестве аномалий на особь представлены в виде средних арифметических со стандартными ошибками среднего. Встречаемость аномалий в выборке и частота встречаемости аномальных особей сравнивались через таблицы сопряженности $2 * 2$ с оценкой $\chi^{2}$ при $p<0,01$. Оценка различий в количестве аномалий на особь исследовалось методом Крускала-Уоллеса. Результаты анализа частоты встречаемости аномальных особей и морфологических аномалий представлены в виде средних арифметических с доверительными интервалами. Расчет доверительных интервалов по частоте встречаемости на разных станциях выполнен методом Уилсона.

\section{Результаты исследования}

При тотальном осмотре скелетов молоди рыб из р. Качи в общей сложности диагностировано 95 морфологических аномалий, принадлежащих к 12 типам.

У молоди пескаря отмечено 62 аномалии, представленные 7 типами. Аномалии локализовались преимущественно в туловищном и хвостовом отделах позвоночного столба. Основную массу составили такие нарушения, как деформация невральных и гемальных дуг хвостового отдела осевого скелета, деформация невральных дуг туловищного отдела осевого скелета. Аномалии позвоночника проявлялись в виде незамкнутых невральных и гемальных дуг, сращения гемальных и невральных дуг разных позвонков, перемещения оснований невральных и гемальных дуг на соседний позвонок, наличия дополнительных ветвей невральных дуг, сращений центров хвостовых позвонков. Также единично отмечены искривление ребер и недоразвитие лучей анального плавника. Число аномалий на особь варьировало от 1,1 до 6,5 и увеличивалось от верховьев к устью. Встречаемость аномалий изменялась в пределах от 6,5 до $55,7 \%$ и также характеризовалась увеличением доли аномалий в выборке в нижнем течении реки. Встречаемость аномальных особей составляла 4,4-10,3\% с максимальным значением на станции 2, минимальным - на станции 3 (табл. 4). Примечательны находки двух особей пескаря, подверженных многочисленным нарушениям осевого скелета (23 и 10 аномалий на особь), на станции 1 (рис. 2). 
Т а б л и ц а 4 [Table 4]

Показатели встречаемости аномалий скелета у молоди рыб из р. Качи [Occurrence indicators of skeletal abnormalities in juvenile fish from the Kacha river]

\begin{tabular}{|c|c|c|c|c|c|c|c|c|}
\hline \multirow{2}{*}{$\begin{array}{c}\text { Аномалия } \\
\text { [Abnormality] }\end{array}$} & \multicolumn{2}{|c|}{$\begin{array}{c}\text { Станция 1 } \\
\text { [Station 1] }\end{array}$} & \multicolumn{2}{|c|}{$\begin{array}{c}\text { Станция 2 } \\
\text { [Station 2] }\end{array}$} & \multicolumn{2}{|c|}{$\begin{array}{l}\text { Станция 3 } \\
\text { [Station 3] }\end{array}$} & \multicolumn{2}{|c|}{$\begin{array}{c}\text { Станция } 4 \\
\text { [Station 4] }\end{array}$} \\
\hline & $S_{\mathrm{ap}}$ & $A_{\mathrm{r}} \%$ & $S_{\text {ap }}$ & $A_{\mathrm{r}} \%$ & $S_{\text {ap }}$ & $A_{\mathrm{r}} \%$ & $S_{\text {ap }}$ & $A_{\mathrm{r}} \%$ \\
\hline \multicolumn{9}{|c|}{ Gobio gobio cynocephalus } \\
\hline B3 & 8 & 20,51 & $\begin{array}{l}9 \\
\end{array}$ & 45,00 & - & - & - & - \\
\hline B8 & 1 & 2,56 & - & - & 3 & 100 & - & - \\
\hline $\mathrm{C} 1$ & - & - & 1 & 5,00 & - & - & - & - \\
\hline $\mathrm{C} 2$ & 1 & 2,56 & - & - & - & - & - & - \\
\hline $\mathrm{C} 3$ & 16 & 41,03 & 6 & 30,00 & - & - & - & - \\
\hline $\mathrm{C} 4$ & 11 & 28,21 & 4 & 20,00 & - & - & - & - \\
\hline F5 & 1 & 2,56 & - & - & - & - & - & - \\
\hline $\begin{array}{c}\text { Количество аномалий, шт. } \\
\text { [Number of abnormalities] }\end{array}$ & \multicolumn{2}{|c|}{39} & \multicolumn{2}{|c|}{20} & \multicolumn{2}{|c|}{3} & \multicolumn{2}{|c|}{-} \\
\hline $\begin{array}{c}\text { Встречаемость } \\
\text { аномальных особей } \\
\text { [Occurrence of abnormal individuals], \% }\end{array}$ & \multicolumn{2}{|c|}{8,57} & \multicolumn{2}{|c|}{10,30} & \multicolumn{2}{|c|}{4,35} & \multicolumn{2}{|c|}{-} \\
\hline $\begin{array}{c}\text { Встречаемость аномалий } \\
\text { [Occurrence of abnormalities], \% }\end{array}$ & \multicolumn{2}{|c|}{55,71} & \multicolumn{2}{|c|}{29,41} & \multicolumn{2}{|c|}{6,52} & \multicolumn{2}{|c|}{-} \\
\hline $\begin{array}{c}\text { Доля тяжелых аномалий, } \\
\text { [Proportion of severe abnormalities], \% }\end{array}$ & \multicolumn{2}{|c|}{82,05} & \multicolumn{2}{|c|}{60,00} & \multicolumn{2}{|c|}{0,00} & \multicolumn{2}{|c|}{-} \\
\hline $\begin{array}{c}\text { Число аномалий на особь, шт. } \\
\text { [Number of abnormalities per } \\
\text { individual], } M \pm m\end{array}$ & \multicolumn{2}{|c|}{$6,5 \pm 3,59$} & \multicolumn{2}{|c|}{$3,3 \pm 1,94$} & \multicolumn{2}{|c|}{$1,14 \pm 0,50$} & \multicolumn{2}{|c|}{-} \\
\hline \multicolumn{9}{|c|}{ Phoxinus phoxinus } \\
\hline B3 & - & - & 2 & 22,20 & 1 & 12,5 & 2 & 18,2 \\
\hline B8 & - & - & - & - & 3 & 37,5 & - & - \\
\hline $\mathrm{C} 2$ & - & - & - & - & - & - & 2 & 18,2 \\
\hline $\mathrm{C} 3$ & 5 & 100 & 5 & 55,60 & - & - & - & - \\
\hline $\mathrm{C} 4$ & - & - & 2 & 22,20 & 1 & 12,5 & 4 & 36,4 \\
\hline D1 & - & - & - & - & - & - & 1 & 9,1 \\
\hline $\mathrm{D} 2$ & - & - & - & - & 2 & 25,0 & - & - \\
\hline D4 & - & - & - & - & - & - & 1 & 9,1 \\
\hline G6 & - & - & - & - & 1 & 12,5 & - & - \\
\hline $\mathrm{I} 7$ & - & - & - & - & - & - & 1 & 9,1 \\
\hline $\begin{array}{c}\text { Количество аномалий, шт } \\
\text { [Number of abnormalities] }\end{array}$ & \multicolumn{2}{|c|}{5} & \multicolumn{2}{|c|}{9} & & 8 & & 11 \\
\hline $\begin{array}{l}\text { Встречаемость аномальных особей } \\
\text { [Occurrence of abnormal individuals], \% }\end{array}$ & & 70 & & 30 & & 36 & &, 20 \\
\hline $\begin{array}{c}\text { Встречаемость аномалий } \\
\text { [Occurrence of abnormalities], \% }\end{array}$ & & 41 & & 00 & & 42 & &, 50 \\
\hline $\begin{array}{c}\text { Доля тяжелых аномалий } \\
\text { [Proportion of severe abnormalities], \% }\end{array}$ & & 00 & & 56 & & 00 & & 09 \\
\hline $\begin{array}{c}\text { Число аномалий на особь, шт. } \\
\text { [Number of abnormalities per } \\
\text { individual], } M \pm m\end{array}$ & & 1,50 & & 9 & 1,5 & $=0,14$ & 1,22 & $\pm 0,15$ \\
\hline
\end{tabular}

Примечание. $S_{\text {ap }}$-общий спектр аномалий; $A_{\mathrm{r}}$-относительная встречаемость аномалий. [Note. $S_{\text {ap }}$ - Total spectrum of abnormalities; $A_{\mathrm{r}}$ - Relative occurrence of abnormalities] 


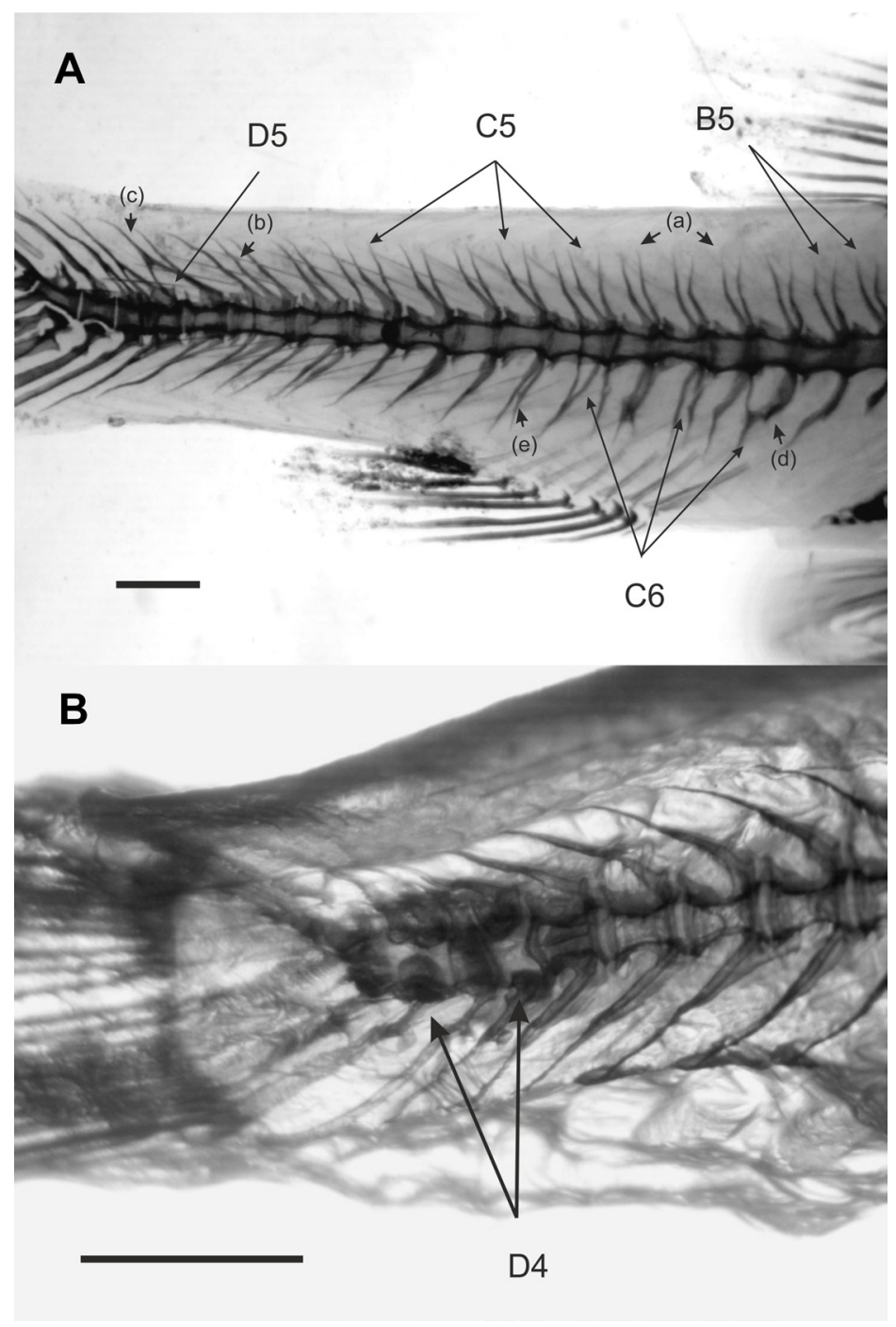

Рис. 2. Аномалии скелета у молоди пескаря сибирского $(A)$ и гольяна речного $(B)$ : аномалии невральных дуг туловищных (B5); хвостовых (С5) ( $a$ - незамкнутые невральные дуги, $b$ - дополнительные ветви невральной дуги) и преуральных позвонков (D5) ( $c$ - сращение невральных дуг разных позвонков); деформация гемальных дуг гемального отдела (C6) $(d$ - сращение гемальных дуг разных позвонков, $e-$ незамкнутые гемальные дуги); деформация тел позвонков хвостового отдела (D4). Размер масштабной линейки 1 мм. Фото автора

[Fig. 2. Skeletal abnormalities in juveniles of the Siberian gudgeon (A) and the common minnow (B): abnormalities of neural arches in prehemal (B5), hemal (C5) ( $a$ - Unclosed neural arches, $b$ - Additional branches of the neural arches) and caudal regions (D5) ( $c$ - Fusion of neural arches of different vertebrae), deformation of hemal arches in hemal region (C6) ( $d$ - Fusion of hemal arches of different vertebrae, unclosed hemal arches), vertebral body deformation in caudal region (D4). Scale bar $=1 \mathrm{~mm}$. Photo is made by NO Yablokov] 
У молоди гольяна диагностировано 33 аномалии, принадлежащие к 10 типам. В целом преобладали нарушения туловищного и хвостового отделов осевого скелета. Морфологические аномалии позвоночника проявлялись в виде деформации и сращения центров позвонков, присутствия незамкнутых невральных и гемальных дуг, перемещения оснований невральных дуг на соседний позвонок. Также отмечены единичные случаи «мопсовидности» и деформации гипуралий. Количественно преобладали такие нарушения, как деформация невральных дуг туловищного отдела, деформация невральных и гемальных дуг хвостового отдела позвоночника. Наибольшее число аномалий на особь составило 9 аномалий, при средневыборочных значениях 1,2-9,0. Встречаемость аномалий в выборках варьировала от 8,4 до 75,0\%. Наибольшие значения встречаемости наблюдались на станции 2, наименьшие - на станции 3. Встречаемость аномальных особей изменялась незначительно в пределах 7,4-11,7\%.

Доли тяжелых форм аномалий у молоди двух видов были близки и варьировали в пределах от 0 до 80\%. Наибольшее число тяжелых аномалий отмечалось на приустьевом участке реки, в то время как в верхней и средней частях реки доля рыб с тяжелыми аномалиями составляла менее чем $10 \%$.

Оценка значимости различий между встречаемостью аномалий на четырех станциях, выполненная посредством критерия $\chi^{2}$, показала наличие статистически значимых различий как для молоди пескаря, так и для молоди гольяна. При уровне значимости $p<0,01$ значения критерия $\chi^{2}$ составили 30,952 у пескаря (при $\left.\chi_{\text {крит }}^{2}=9,21\right)$ и 39,051 у гольяна (при $\left.\chi_{\text {крит }}^{2}=11,345\right)$. При оценке доверительных интервалов для частоты встречаемости аномалий на трех изученных станциях обнаружены различия между выборками молоди пескаря. У молоди гольяна обнаружены статистически значимые различия между станцией 2 и станциями 3 и 4 (рис. $3, A$ ).

При аналогичной оценке значимости различий между встречаемостью аномальных особей различия не отмечены (рис. $3, B$ ).

При оценке различий в количестве аномалий на особь у молоди рыб, отобранной с четырех исследованных станций, различия не обнаружены. Значимые различия в количестве аномалий между видами также отсутствовали.

\section{Обсуждение результатов исследования}

Анализ количественных и качественных характеристик морфологических аномалий скелета у молоди пескаря сибирского и гольяна речного из р. Качи продемонстрировал значительные расхождения по ряду показателей в различных участках реки.

В частности, при анализе встречаемости аномалий в р. Каче прослеживается тенденция увеличения частоты встречаемости морфологических аномалий скелета от верхнего участка реки к нижнему. Наибольшим числом аномалий характеризуются рыбы с участков реки, расположенных в черте г. 
Красноярска и ниже д. Дрокино, чего нельзя сказать о частоте встречаемости аномальных особей. Колебания в частоте встречаемости аномальных особей изменяются в пределах от 4,6 до 10,4\% для молоди пескаря и от 7,4 до 11,7\% для молоди гольяна. Однако, согласно работам В.С. Кирпичникова, в естественных популяциях рыб, не испытывающих значительной антропогенной нагрузки, процент аномальных особей не должен превышать 5\% [22]. Полученные значения встречаемости аномальных особей, за исключением молоди пескаря, обитающей в районе пос. Памяти 13 Борцов, в полтора-два раза выше допустимой величины.
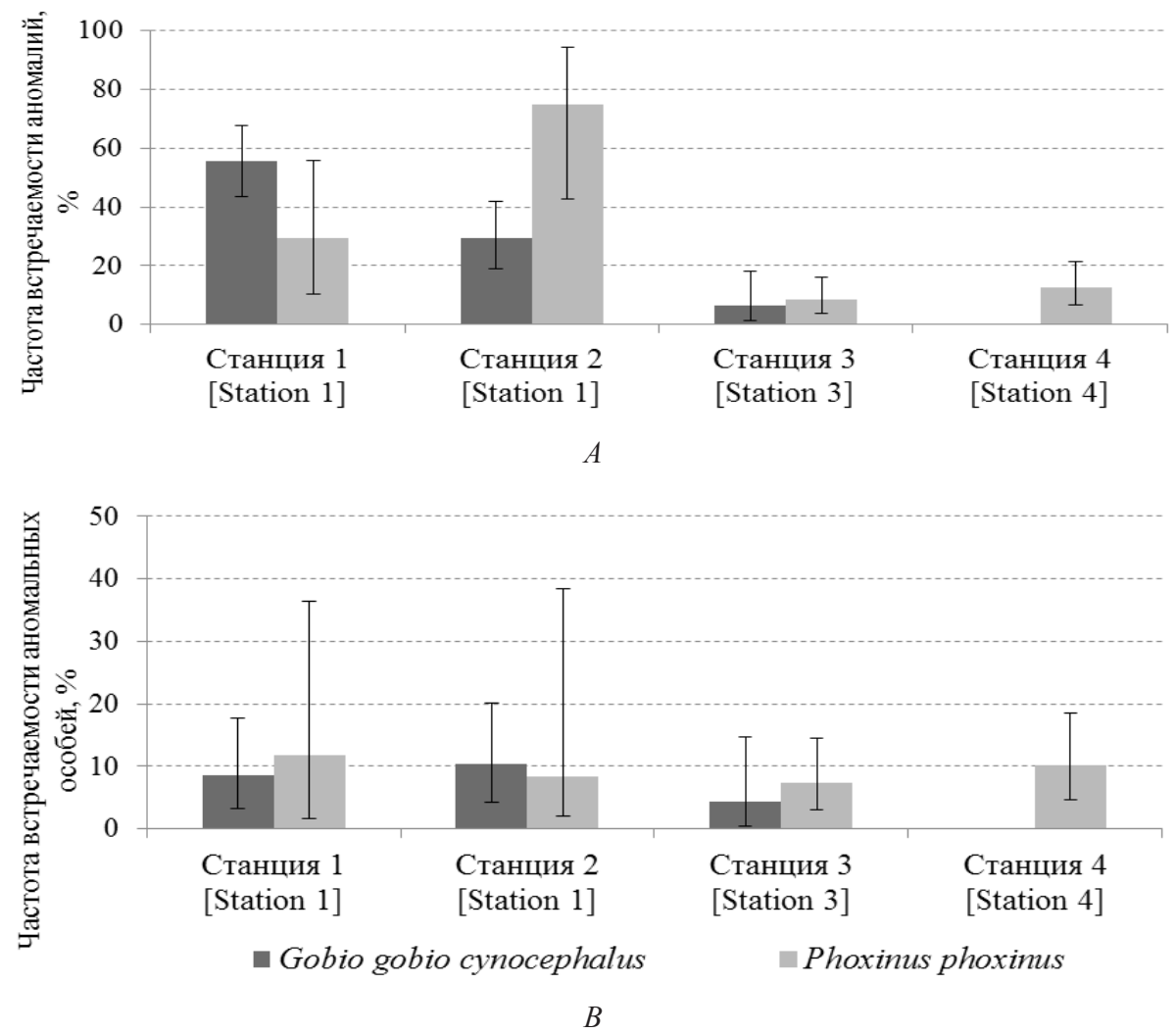

Рис. 3. Частота встречаемости аномалий $(A)$ и аномальных особей $(B)$ у молоди рыб из р. Качи. Планки погрешностей представлены в виде доверительных интервалов по Уилсону

[Fig. 3. Frequency of abnormalities (A) and abnormal individuals (B) in juvenile fish from the Kacha river. On the X-axis - Sampling sites; on the Y-axis Occurrence of abnormalities (A) and occurrence of abnormal individuals (B). Error bars are presented as Wilson confidence intervals] 
Также следует отметить, что значения показателей встречаемости аномалий в развитии скелета в нижнем течении р. Качи близки к частоте встречаемости аномалий позвоночного столба у молоди рыб, населяющих приустьевые участки некоторых пригородных водотоков г. Красноярска, подверженных антропогенной нагрузке [23].

При качественной оценке отмеченных аномалий стоит обратить внимание на большое количество тяжелых форм аномалий в нижних участках реки, составляющих 80-50\% от общего числа аномалий. Присутствие данных групп аномалий в естественных популяциях наиболее критично, так как напрямую связано с жизнеспособностью рыбы.

Диагностированные в пробах виды аномалий преимущественно представляют собой разнообразные деформации невральных и гемальных дуг, такие как незамкнутые дуги позвонков, сращение дуг разных позвонков, перемещение дуг на соседние позвонки. Аномалии локализовались, прежде всего, в хвостовом отделе позвоночника и, в меньшей степени, в туловищном отделе.

Схожие группы аномалий, в частности незамкнутые дуги позвонков, сращение дуг разных позвонков, наличие дополнительных невральных дуг, перемещение дуг на соседние позвонки, отмечались Ю.В. Чеботаревой у сеголеток плотвы Rutilus rutilus (L., 1758) при экспериментальном воздействии на икру малых доз хлорофоса и $\mathrm{N}$-метил-N'-нитро-N-нитрозогуадина (MNNG) [12]. Множественные аномалии позвоночного столба, такие как сращения тел и дуг позвонков, незамкнутые дуги позвонков, наличие дополнительных невральных дуг, перемещение дуг на соседние позвонки, с частотой встречаемости 94-97\% отмечались И.Г. Богуцкой с соавт. у плотвы в окрестных водных объектах производственного объединения «Маяк» [19]. Аномалии морфологии позвонков регистрируются у взрослых особей и молоди тиляпии Oreochromis spp. в нескольких загрязненных водотоках на юго-западе Тайваня [24]. Такие нарушения, как мопсовидность и сращение центров позвонков, отмечались у половозрелых особей сиговых в озерах Норило-Пясинской водной системы, в зоне воздействия Норильского горно-металлургического комбината [25], а также у леща Abramis brama (L., 1758) в двух техногенно загрязненных водных объектах в бассейне Рейна [26]. Л.И. Соколов (1998) отмечает мопсовидность и искривление позвоночника у представителей ихтиофауны р. Москвы: плотвы, серебряного карася Carassius gibelio (Bloch, 1782), леща, судака Sander lucioperca (L., 1758), окуня Perca fluviatilis L., 1758 [27]. Аналогичные аномалии диагностированы у молоди окуня в оз. Ильменском [11].

В настоящее время известно множество примеров ингибирования поглощения кальция при высоких содержаниях в воде кадмия, свинца, цинка, стронция, алюминия [28-29]. В ряде работ ионы алюминия и стронция отмечаются в качестве факторов, индуцирующих нарушения кальцификации скелета, в дальнейшем проявляющиеся в виде искривлений позвоночника и 
остеопороза [30-31]. Нарушение химического состава костной ткани, возникновение нарушений в строении осевого скелета также регистрируются у рыб, подверженных влиянию различных хлорорганических пестицидов [32-33].

Список поллютантов в р. Каче включает широкий спектр веществ органической и неорганической природы. Очевидно, что такое многообразие загрязняющих агентов служит причиной различных структурных и функциональных изменений в сообществе рыб, в том числе и возникновения морфологических нарушений в процессах индивидуального развития. Подобные случаи хорошо известны для ряда пригородных водных объектов в нашей стране и за рубежом [24, 27, 34].

Таким образом, велика вероятность того, что высокая встречаемость морфологических аномалий в нижнем течении р. Качи связана именно с воздействием определенных групп токсикантов. Однако в связи с разнообразием загрязняющих веществ в реке и недостаточной изученностью механизмов формирования различных форм отклонений развития выявление конкретного фактора воздействия на онтогенез рыб на данном этапе не представляется возможным.

\section{Заключение}

Проведена количественная и качественная оценка морфологических аномалий скелета у молоди пескаря сибирского и гольяна речного, обитающих в р. Каче. Всего обнаружено 95 аномалий, принадлежащих к 12 типам и локализованных в 6 отделах скелета. Среди них у молоди пескаря - 62 аномалии 7 типов, у молоди гольяна - 33 аномалии 10 типов. При анализе частот встречаемости аномалий в р. Каче прослеживается тенденция увеличения встречаемости морфологических аномалий скелета от верхнего участка реки к нижнему по мере роста техногенного воздействия на водоток. Значения показателей встречаемости аномалий в нижнем течении р. Качи близки к частотам встречаемости аномалий позвоночного столба у молоди рыб, ранее отмеченным для некоторых пригородных водотоков г. Красноярска, подверженных антропогенной нагрузке.

\section{Лuтература}

1. Ткачев Б.П., Булатов В.И. Малые реки: экологическое состояние и экологические проблемы. Новосибирск : ГПНТБ СО РАН, 2002. Вып. 64. 114 с. (Серия: Экология).

2. Селезнева А.В. Антропогенная нагрузка на реки от точечных источников загрязнения // Известия Самарского научного центра РАН. 2003. Т. 5, № 2. С. 268-277.

3. О состоянии и охране окружающей среды Российской Федерации в 2015 году: Государственный доклад. М. : Минприроды России, 2016. 639 с.

4. О состоянии и охране окружающей среды Российской Федерации в 2014 году: Государственный доклад. М. : Минприроды России, 2015. 473 с. 
5. О состоянии и охране окружающей среды в Красноярском крае в 2015 году: Государственный доклад. Красноярск, 2016. 314 с.

6. Моисеенко Т.И., Гашев С.Н., Селюков А.Г., Жигилева О.Н., Алешина О.Н. Биологические методы оценки качества вод. Ч. 1: Биоиндикация // Вестник Тюменского государственного университета. Экология и природопользование. 2010. № 7. C. $20-40$.

7. Попов П.А. Оценка экологического состояния водоемов методами ихтиоиндикации. Новосибирск : НГУ, 2002. 270 с.

8. Кашулин Н.А., Лукин А.А., Амундсен П.А. Рыбы пресных вод Субарктики как биоиндикаторы техногенного загрязнения. Апатиты : Изд-во Кольского научного центра АН СССР, 1999. 142 с.

9. Евланов И.А., Минеев А.К., Розенберг Г.С. Оценка состояния пресноводных экосистем по морфологическим аномалиям у личинок рыб. Тольятти : ИЭВБ РАН, 1999. 38 с.

10. Госькова О.А., Мельниченко И.П., Богданов В.Д. Морфологические аномалии и травмы у пеляди в период нерестовой миграции в уральских притоках Оби // Вестник АГТУ. Сер. Рыбное хозяйство. 2014. № 4. С. 7-15.

11. Перескоков А.В. Сравнение уровня аберраций у молоди окуня с накоплением тяжелых металлов в икре // Известия Челябинского научного центра. 2004. №. 3. С. 101-105.

12. Чеботарева Ю.В. Аномалии в строении позвоночника у сеголеток плотвы Rutilus rutilus после воздействия токсикантов на ранние стадии развития // Вопросы ихтиологии, 2009. Т. 49, № 1. С. 102-110.

13. Boglione C., Costa C., Giganti M., Cecchetti M., Di Dato P., Scardi M., Cataudella S. Biological monitoring of wild thicklip grey mullet (Chelon labrosus), golden grey mullet (Liza aurata), thinlip mullet (Liza ramada) and flathead mullet (Mugil cephalus) (Pisces: Mugilidae) from different Adriatic sites: meristic counts and skeletal abnormalities // Ecological Indicators. 2006. № 6. PP. 712-732.

14. Минеев А.К. Неспецифические реакции у рыб из водоемов Средней и Нижней Волги // Известия Самарского научного центра Российской академии наук. 2013. Т. 15, № 3 (7). С. 2301-2318.

15. Гольд 3.Г., Глущенко Л.А., Морозова И.И., Шулепина С.П., Шадрин И.А., Глазков А.Е. Качество воды реки Кача по биологическим (перифитон, макрозообентос, биотестирование) и химическим дескрипторам // Вестник КрасГУ. 2005. Вып. 5. C. $137-146$.

16. Семенова Е.М. Донные сообщества в оценке качества воды реки Кача // Экология Южной Сибири и сопредельных территорий : материалы XIV Междунар. научнопрактической школы-конференции студентов и молодых ученых / под ред. В.В. Анюшина. Абакан : ХГУ им. Н. Ф. Катанова, 2010. Вып. 14, т. 1. С. 89-90

17. Potthoff T. Clearing and staining techniques // Ontogeny and systematics of fishes. Special Publication of American Society of Ichthyology. Moser H.G., editor. Lawrence : Allen Press, 1984. Vol. 1. PP. 35-37.

18. Naseka A.M. Comparative study on the vertebral column in the Gobioninae (Cyprinidae, Pisces) with special reference to its systematics // Publicaciones Especiales Instituto Espanol de Oceanografia. 1996. Vol. 21. PP. 149-167.

19. Bogutskaya N.G., Zuykov M.A., Naseka A.M., Anderson E.B. Normal axial skeleton structure in common roach Rutilus rutilus (Actinopterygii: Cyprinidae) and malformations due to radiation contamination in the area of the Mayak (Chelyabinsk Province, Russia) nuclear plant // Journal of fish biology. 2011. Vol. 79, № 4. PP. 991-1016.

20. Boglione C., Gagliardi F., Scardi M., Cataudella S. Skeletal descriptors and quality assessment in larvae and post-larvae of wild-caught and hatchery-reared gilthead sea bream (Sparus aurata L. 1758) // Aquaculture. 2002. № 192. PP. 1-22. 
21. Боркин Л.Я., Безман-Мосейко О.С., Литвинчук С.Н. Оценка встречаемости морфологических аномалий в природных популяциях (на примере амфибий) // Труды Зоологического института РАН. 2012. Т. 316, №. 4. С. 324-343.

22. Кирпичников В.С. Генетика и селекция рыб. Л. : Наука, 1987. 320 стр.

23. Яблоков Н.О. Разнообразие и встречаемость морфологических аномалий молоди рыб водных объектов Енисея и Оби // Биоэкологическое краеведение: мировые, российские и региональные проблемы : материалы II Всероссийской научнопрактической конференции с международным участием / под ред. Ю. М. Попова. Самара : Порто-принт, 2013. С. 303-310.

24. Sun P.L., Hawkins W.E., Overstreet R.M., Brown-Peterson N.J. Morphological deformities as biomarkers in fish from contaminated rivers in Taiwan // International journal of environmental research and public health. 2009. Vol. 6, № 8. PP. 2307-2331.

25. Савваитова К.А., Чеботарева Ю.В., Пичугин М.Ю., Максимов С.В. Аномалии в строении рыб как показатели состояния природной среды // Вопросы ихтиологии. 1995. T. 35, № 2. C. 182-188.

26. Slooff W. Skeletal abnormalities in fish from polluted surface waters // Aquatic toxicology. 1982. Vol. 2, № 3. PP. 157-173.

27. Соколов Л.И. Рыбы в условиях мегаполиса (г. Москва) // Соросовский образовательный журнал. 1998. № 5. С. 30-35.

28. Моисеенко Т.И. Водная токсикология: фундаментальные и прикладные аспекты. М. : Наука, 2009. 400 с.

29. Sfakianakis D.G., Renieri E., Kentouri M., Tsatsakis A.M. Effect of heavy metals on fish larvae deformities : a review // Environmental research. 2015. Vol. 137. PP. 246-255.

30. Moiseenko T.I., Kudryavtseva L.P. Trace metal accumulation and fish pathologies in areas affected by mining and metallurgical enterprises in the Kola Region, Russia // Environmental Pollution. 2001. Vol. 114, № 2. PP. 285-297.

31. Rosseland B.O., Staurnes M. Physiological mechanisms for toxic effects and resistance to acidic water: an ecophysiological and ecotoxicological approach // Acidification of freshwater ecosystems: implications for the future. 1994. Vol. 14. PP. 227-245.

32. Брагинский Л.П., Комаровский Ф.Я., Мережко А.И. Персистентные пестициды в экологии пресных вод. Киев: Наукова думка, 1979. 143 с.

33. Mehrle P.M., Mayer J.F.L. Toxaphene effects on growth and bone composition of fathead minnows, Pimephales promelas // Journal of the Fisheries Board of Canada. 1975. Vol. 32, № 5. PP. 593-598.

34. Минеев А.К., Калинин Е.А. Видовой состав и морфологические аномалии молоди рыб из двух малых рек Удмуртской республики // Вестник Удмуртского университета. Сер. Биология. Науки о земле. 2013. Вып. 1. С. 92-98

Поступила в редакиию 08.06.2017 г.; повторно 18.08.2017 г.; принята 27.02.2018 г.; опубликована 30.03.2018 г.

Яблоков Никита Олегович - м.н.с. Научно-исследовательского института экологии рыбохозяйственных водоемов (Россия, 660049, г. Красноярск, ул. Парижской коммуны, 33).

E-mail: evilapple@mail.ru

For citation: Yablokov NO. Morphological abnormalities in the skeleton of juvenile fish from the Kacha river (Middle Yenisei system) in the gradient of anthropogenic impact. Vestnik Tomskogo gosudarstvennogo universiteta. Biologiya = Tomsk State University Journal of Biology. 2018;41:156-173. doi: 10.17223/19988591/41/9 In Russian, English Summary 


\section{Nikita O. Yablokov}

Research Institute of Ecology of Fishery Reservoirs, Krasnoyarsk, Russian Federation

\section{Morphological abnormalities in the skeleton of juvenile fish from the Kacha river (Middle Yenisei system) in the gradient of anthropogenic impact}

The predominant part of small rivers of Russia is subject to active technogenic impact. The most critical is manifested in urban and suburban water bodies, taking on a huge number of organic and inorganic pollutants. The striking example of such impact in the Yenisei river basin is the Kacha river $\left(64^{\circ} 00^{\prime} 58^{\prime \prime} \mathrm{N}, 92^{\circ} 53^{\prime} 32^{\prime \prime} \mathrm{E}\right)$, the lower course of which is located within the city of Krasnoyarsk and constantly exposed to technogenic pollutants. The intensive technogenic load, as a rule, negatively affects the communities of hydrobionts, including juvenile fish, which have the lowest level of toxic resistance in comparison with adults and are also unable to actively leave areas with high levels of pollution. One of the responses of the fish community to the effect of a complex of unfavorable factors caused by the anthropogenic load on water bodies, including the presence of toxic substances in the ecosystem can be the emergence of various morphological abnormalities. This fact makes it possible to use the indicators of occurrence and diversity of morphological abnormalities as criteria for water quality assessment.

The aim of this research was to evaluate the diversity and the occurrence of skeletal abnormalities in juvenile Siberian gudgeon Gobio gobio cynocephalus Dybowski, 1869 and common minnow Phoxinus phoxinus (L., 1758) from the Kacha river (Middle Yenisei system). The material for the study was selected in July-August 2016, at four sites of the river differing in the level of technogenic pressure and the spectrum of pollutants (See Fig.1). The list of the main polluting agents, as well as the summary information on the environmental monitoring results at the investigated sites of the river are given in Table 1. In total, 184 Siberian gudgeons and 212 common minnows were examined. The size and age characteristics of the studied individuals are shown in Table 2. Abnormalities were studied in specimens previously cleared of soft tissues and stained with alizarin red according to the Potthoff method with a $40 \times$ increase. The examination was carried out in 9 parts of the skeleton, successively (See Table 3). Nomenclature and differentiation of abnormalities in terms of severity are given according to Chebotareva (2009) and Boglione et al (2002). To describe the type and location of the abnormality, a system of symbols was used according to which each part of the skeleton was assigned an alphabetical index; a particular abnormality type got a numerical index (See Table 3). The frequency of abnormal specimens, the total spectrum of abnormalities, the frequency of abnormalities, the relative occurrence of abnormalities, the number of abnormalities in an individual, and the load of severe abnormalities were studied. The incidence of abnormalities and occurrence of abnormal specimens were compared using contingency tables $2 \times 2$ with an estimate by Chisquared test with $\mathrm{p}<0.01$. Confidence intervals for occurrence at different sites were calculated using Wilson method. Differences in the number of abnormalities were investigated by the Kruskal-Wallis test.

In total, 95 abnormalities belonging to 12 types were detected. Among them, 62 abnormalities belonging to 7 types in juvenile gudgeons and 33 abnormalities belonging to 10 types in juvenile minnows were identified (See Tables). Abnormalities were localized predominantly in the abdominal part of the vertebral spine and presented a variety of neural and hemal arch deformation, such as unclosed vertebral arches, fusion of vertebral arches and displacement of arches to adjacent vertebrae. The incidence of 
abnormalities varied from 6.5 to $55.7 \%$ in juvenile gudgeons and from 8.4 to $75.0 \%$ in juvenile minnows. The incidence of abnormal specimens was $4.6-10.4 \%$ for gudgeons and $7.4-11.7 \%$ for minnows. Proportions of severe abnormalities in juveniles of two species were similar and ranged from 80 to $0 \%$ from the upper reaches of the river to the lower. When analyzing the occurrence of abnormalities in the Kacha river, a tendency to increase the frequency of occurrence was witnessed as the anthropogenic load on the watercourse increased. In general, the occurrence of skeletal abnormalities in the Kacha River lower reaches was close to the occurrence of the vertebral spine abnormalities in juvenile fish, previously noted in a few suburban waterways near Krasnoyarsk.

The paper contains 3 Figures, 4 Tables and 34 References.

Key words: fish ontogenesis; morphological abnormalities; technogenic influence; Phoxinus phoxinus; Gobio gobio cynocephalus.

\section{References}

1. Tkachev BP, Bulatov VI. Malye reki: ekologicheskoe sostoyanie i ekologicheskie problemy [Small rivers: state-of-the act and ecological problem]. Analytical Review. Ser. Ecology. Vol. 64. Novosibirsk: State Public Scientific and Technological Library of the SB RAS Publ.; 2002; 114 p. In Russian

2. Selezneva AV. Antropogenic load on rivers by point springs of pollution. Izvestia of Samara Scientific Center of the Russian Academy of Sciences. 2003;5(2):268-277. In Russian

3. Gosudarstvennyy doklad «O sostoyanii $i$ okhrane okruzhayushchey sredy Rossiyskoy Federatsii v 2015 godu» [State report "On the state of the environment and its protection in the Russian Federation in 2015"]. Moscow: Ministry of Natural Resources and Environment of Russia Publ.; 2016. 639 p. In Russian

4. Gosudarstvennyy doklad «O sostoyanii $i$ okhrane okruzhayushchey sredy Rossiyskoy Federatsii v 2014 godu» [State report "On the state of the environment and its protection in the Russian Federation in 2014"]. Moscow: Ministry of Natural Resources and Environment of Russia Publ.; 2015. 473 p. In Russian

5. Gosudarstvennyy doklad "O sostoyanii i okhrane okruzhayushchey sredy v Krasnoyarskom krae v 2015 godu» [State report "On the state of the environment and its protection in Krasnoyarsk Krai in 2015"]. Krasnoyarsk: Ministry of Natural Resources and Environment of Krasnoyarsk Krai Publ.; 2016. 314 p. In Russian

6. Moiseyenko TI, Gashev SN, Selyukov AG, Zhigileva ON, Aleshina ON. Biological methods of waters quality estimation: Part 1. Bioindication. Tyumen State University Herald. Natural Resource Use and Ecology. 2010;7:20-40. In Russian

7. Popov PA. Otsenka ekologicheskogo sostoyaniya vodoemov metodami ikhtioindikatsii Assessment of the ecological state of water bodies by the methods of ichtyoindication]. Novosibirsk: Novosibirsk State University Publ.; 2002. 267 p. In Russian

8. Kashulin NA, Lukin AA, Amundsen PA. Ryby presnykh vod Subarktiki kak bioindikatory tekhnogennogo zagryazneniya [Freshwater fish of the Subarctic as bioindicators of technogenic pollution]. Apatity: Kola Science Centre of the Academy of Sciences of the USSR Publ.; 1999. 142 p. In Russian

9. Evlanov IA, Mineev AK, Rozenberg GS. Otsenka sostoyaniya presnovodnykh ekosistem po morfologicheskim anomaliyam u lichinok ryb [Freshwater ecosystem health assessment according to morphological abnormalities in fish larvae]. Tolyatti: Institute of Ecology of the Volga baisin RAS Publ.; 1999. 38 p. In Russian

10. Goskova OA, Melnichenko IP, Bogdanov VD. Morphological anomalies and traumas of peled during spawning migration in the Ural Ob tributaries. Vestnik of Astrakhan State Technical University. Series: Fishing Industry. 2014;4:7-15. In Russian 
11. Pereskokov AV. Sravnenie urovnya aberratsiy u molodi okunya s nakopleniem tyazhelykh metallov $\mathrm{v}$ ikre [Comparison of the level of aberrations in juvenile perch with heavy metal accumulation in caviar]. Izvestiya Chelyabinskogo nauchnogo tsentra URO RAN. 2004;3:101-105. In Russian

12. Chebotareva YuV. Abnormalities in the backbone structure in roach (Rutilus rutilus) (Cyprinidae, Cepriniformes) underyearlings following toxic impacts during early developmental stages. Journal of Ichthyology. 2009;49(1):96-104. doi: 10.1134/ S0032945209010123

13. Boglione C, Costa C, Giganti M, Cecchetti M, Di Dato P, Scardi M, Cataudella S. Biological monitoring of wild thicklip grey mullet (Chelon labrosus), golden grey mullet (Liza aurata), thinlip mullet (Liza ramada) and flathead mullet (Mugil cephalus) (Pisces: Mugilidae) from different Adriatic sites: meristic counts and skeletal abnormalities. Ecological Indicators. 2006;6:712-732. doi: 10.1016/j.ecolind.2005.08.032

14. Mineev AK. Nonspecific reactions in fish from waters Middle and Lower Volga. Izvestia of Samara Scientific Center of the Russian Academy of Sciences. 2013;15(3(7)):2301-2318. In Russian

15. Gold ZG, Glushchenko LA, Morozova II, Shulepina SP, Shadrin IA, Glazkov AE. Kachestvo vody reki Kacha po biologicheskim (perifiton, makrozoobentos, biotestirovanie) i khimicheskim deskriptoram [The Kacha river water quality according to biological (periphyton, macrozoobenthos, biotesting) and chemical descriptors]. Vestnik Krasnoyarskogo gosydarstvennogo universiteta. 2005;5:137-146. In Russian

16. Semenova EM. Donnye soobshchestva v otsenke kachestva vody reki Kacha [Benthic communities in assessing the quality of the Kacha river water]. In: Ekologiya Yuzhnoy Sibiri i sopredel'nykh territoriy. Materialy XIV Mezhdunar. nauchno-prakticheskoy shkolykonferentsii studentov i molodykh uchenykh [Ecology of Southern Siberia and adjacent territories. Proc. of the XIV Int. Sci. Conf. of Students and Young Scientists]. Anyushina VV, editor. Abakan: Khakassian State University Publ.; 2010, 14(1). pp. 89-90. In Russian

17. Potthoff T. Clearing and staining techniques. Ontogeny and systematics of fishes. Special Publication of American Society of Ichthyology. Moser HG, editor. Lawrence: Allen Press; 1984;1:35-37.

18. Naseka AM. Comparative study on the vertebral column in the Gobioninae (Cyprinidae, Pisces) with special reference to its systematic. Publicaciones Especiales Instituto Espanol de Oceanografia. 1996;21:149-167.

19. Bogutskaya NG, Zuykov MA, Naseka AM, Anderson EB. Normal axial skeleton structure in common roach Rutilus rutilus (Actinopterygii: Cyprinidae) and malformations due to radiation contamination in the area of the Mayak (Chelyabinsk Province, Russia) nuclear plant. Journal of Fish Biology. 2011;79(4):991-1016. doi: 10.1111/j.10958649.2011.03078.x

20. Boglione C, Gagliardi F, Scardi M, Cataudella S. Skeletal descriptors and quality assessment in larvae and post-larvae of wild-caught and hatchery-reared gilthead sea bream (Sparus aurata L. 1758). Aquaculture. 2002;192:1-22. doi: 10.1016/S0044-8486(00)00446-4

21. Borkin LJ, Bezman-Moseyko OS, Litvinchuk SN. Evaluation of animal deformity occurrence in natural populations (an example of amphibians. Proceedings of the Zoological Institute of the Russian Academy of Sciences. 2012;316(4):324-343. In Russian

22. Kirpichnikov VS. Genetika i selektsiya ryb [Genetics and selection of fishes]. Leningrad; Nauka Publ.; 1987. 320 p. In Russian

23. Yablokov NO. Raznoobrazie i vstrechaemost' morfologicheskikh anomaliy molodi ryb vodnykh ob"ektov Eniseya i Obi [Diversity and occurrence of morphological abnormalities in juvenile fish from water bodies of the Yenisei and the Ob' rivers]. In: Bioekologicheskoe kraevedenie: mirovye, rossiyskie i regional'nye problemy. Materialy II-y Vserossiyskoy nauchno-prakticheskoy konferentsii s mezhdunarodnym uchastiem [Bioecological local 
studies: World, Russian and regional problems. Proc. of the II All-Russian Sci. Conf. with Int. Part.]. Popova YM, editor. Samara: Porto-print Publ.; 2013. pp. 303-310 In Russian

24. Sun PL, Hawkins WE, Overstreet RM, Brown-Peterson NJ. Morphological deformities as biomarkers in fish from contaminated rivers in Taiwan. International Journal of Environmental Research and Public health. 2009;6(8):2307-2331. doi: 10.3390/ ijerph6082307

25. Savvaitova KA, Chebotareva YuV, Pichugin MYu, Maksimov SV. Anomalii v stroenii ryb kak pokazateli sostoyaniya prirodnoy sredy [Abnormalities in fish anatomy as an indicator of the environmental health]. Journal of Ichthyology. 1995;35(2):182-188. In Russian

26. Slooff W. Skeletal abnormalities in fish from polluted surface waters. Aquatic toxicology. 1982;2(3):157-173. doi: 10.1016/0166-445X(82)90013-3

27. Sokolov LI. Ryby v usloviyakh megapolisa (g. Moskva) [Fish in a megapolis (Moscow)]. Sorosovskiy obrazovatel'nyy zhurnal. 1998;4(5):30-35. In Russian

28. Moiseenko TI. Vodnaya toksikologiya: fundamental'nye i prikladnye aspekty [Water toxicology: Fundamental and applied aspects]. Moskow: Nauka Publ.; 2009. 400 p. In Russian

29. Sfakianakis DG, Renieri E, Kentouri M, Tsatsakis AM. Effect of heavy metals on fish larvae deformities: A review. Environmental Research. 2015;137:246-255. doi: 10.1016/j. envres.2014.12.014

30. Moiseenko TI, Kudryavtseva LP. Trace metal accumulation and fish pathologies in areas affected by mining and metallurgical enterprises in the Kola Region, Russia. Environmental Pollution. 2001;114(2):285-297. doi: 10.1016/S0269-7491(00)00197-4

31. Rosseland BO, Staurnes M. Physiological mechanisms for toxic effects and resistance to acidic water: An ecophysiological and ecotoxicological approach. In: Acidification of Freshwater Ecosystems: Implications for the Future. Steinberg CEW, Wright RF, editors. Chichester: John Wiley \& Sons; 1994;14:227-245.

32. Braginskiy LP, Komarovskiy FYa, Merezhko AI. Persistentnye pestitsidy v ekologii presnykh vod [Persistent pesticides in freshwater ecology]. Kiev: Naukova dumka Publ.; 1979. 143 p. In Russian

33. Mehrle PM, Mayer JFL. Toxaphene effects on growth and bone composition of fathead minnows, Pimephales promelas. Journal of the Fisheries Board of Canada. 1975;32(5):593598. doi: 10.1139/f75-076

34. Mineev AK, Kalinin EA. Species composition and morphological anomalies of young fish from two small rivers of the Udmurtian Republic. Bulletin of Udmurt University. Series Biology. Earth Sciences. 2013;6(1):92-98. In Russian

Received 06 June 2017; Revised 18 August 2017; Accepted 17 January 2018; Published 30 March 2018

\author{
Author info: \\ Yablokov Nikita O, Junior Researcher, Research Institute of Ecology of Fishery Reservoirs, 33 Parizhskoy \\ Komunny Str., Krasnoyarsk 660049, Russian Federation. \\ E-mail: evilapple@mail.ru
}

